\title{
Global Dynamics of an Epidemic Model with a Ratio-Dependent Nonlinear Incidence Rate
}

\author{
Sanling Yuan and Bo Li \\ College of Science, Shanghai University for Science and Technology, Shanghai 200093, China \\ Correspondence should be addressed to Sanling Yuan, sanling@usst.edu.cn
}

Received 14 May 2009; Accepted 21 August 2009

Recommended by Antonia Vecchio

\begin{abstract}
We study an epidemic model with a nonlinear incidence rate which describes the psychological effect of certain serious diseases on the community when the ratio of the number of infectives to that of the susceptibles is getting larger. The model has set up a challenging issue regarding its dynamics near the origin since it is not well defined there. By carrying out a global analysis of the model and studying the stabilities of the disease-free equilibrium and the endemic equilibrium, it is shown that either the number of infective individuals tends to zero as time evolves or the disease persists. Computer simulations are presented to illustrate the results.
\end{abstract}

Copyright (C) 2009 S. Yuan and B. Li. This is an open access article distributed under the Creative Commons Attribution License, which permits unrestricted use, distribution, and reproduction in any medium, provided the original work is properly cited.

\section{Introduction}

In recent years, attempts have been made to develop realistic mathematical models for the transmission dynamics of infectious diseases. In modeling of communicable diseases, the incidence function has been considered to play a key role in ensuring that the models indeed give reasonable qualitative description of the transmission dynamics of the diseases $[1,2]$. Some factors, such as media coverage, density of population, and life style, may affect the incidence rate directly or indirectly [3].

Let $S(t)$ be the number of susceptible individuals, let $I(t)$ be the number of infective individuals, and let $R(t)$ be the number of removed individuals at time $t$. After a study of the cholera epidemic spread in Bari in 1973, Capasso and Serio [4] introduced a saturated incidence rate $g(I) S$ into epidemic models. This is important because the number of effective contacts between infective individuals and susceptible individuals may saturate at high infective levels due to crowding of infective individuals or due to the protection measures by the susceptible individuals. If the function $g(I)$ is decreasing when $I$ is large, it can also be used to interpret the "psychological" effects: for a very large number of 
infectives the infection force may decrease as the number of infective individuals increases because in the presence of large number of infectives the population may tend to reduce the number of contacts per unit time. To incorporate the effect of the behavioral changes of the susceptible individuals, Liu et al. [5,6] used a nonlinear incidence rate given by

$$
g(I)=\frac{k I^{l}}{1+\alpha I^{h}}
$$

where the parameters $k, l$, and $h$ are positive constants and $\alpha$ is a nonnegative constant, $k I^{l}$ measures the infection force of the disease, and $1 /\left(1+\alpha I^{h}\right)$ measures the inhibition effect from the behavioral change of the susceptible individuals when their number increases or from the crowding effect of the infective individuals. The special cases when $l, h$, and $\alpha$ take different values have been used by many authors. See, for example, Derrick and van den Driessche [7], Hethcote [8], Hethcote and Levin [9] and van den Driessche [10], Alexander and Moghadas [11], Ruan and Wang [12], Xiao and Ruan [13], and so forth.

Note that the infectious force $g(I)$ in (1.1) is only a function of infective individuals. However in the transmission of communicable diseases, it involves both infective individuals and susceptible individuals. Thus we think the infectious force ought to depend on the densities of both infective individuals and susceptible individuals and it should take the form $g(I, S)$. Assuming that the infectious force is a function of the ratio of the number of the infectives to that of the susceptibles, in this paper, we consider the following infectious force function:

$$
g(I, S)=g\left(\frac{I}{S}\right)=\frac{k(I / S)^{l}}{1+\alpha(I / S)^{h}}
$$

To describe the psychological or inhibitory effect from the behavioral change of the susceptible individuals when the number of infective individuals is very large, Xiao and Ruan [13] have considered the following SIRS model:

$$
\begin{aligned}
& \frac{d S}{d t}=b-d S-\frac{k I S}{1+\alpha I^{2}}+\gamma R \\
& \frac{d I}{d t}=\frac{k S I}{1+\alpha I^{2}}-(d+\mu) I \\
& \frac{d R}{d t}=\mu I-(d+\gamma) R
\end{aligned}
$$

where the infectious force $g(I)$ takes the following form:

$$
g(I)=\frac{k I}{1+\alpha I^{2}}
$$

which is the special case when $l=1, h=2$ in (1.1). All the parameters are positive numbers. Also $b$ is the recruitment rate of the population, $d$ is the natural death rate of the population, 
$k$ is the proportionality constant, $\gamma$ is the natural recovery rate of the infective individuals, $\mu$ is the rate at which recovered individuals lose immunity and return to the susceptible class, and $\alpha$ is the parameter measures the psychological or inhibitory effect. Global analysis carried out by them shows that either the number of infective individuals tends to zero as time evolves or the disease persists.

Instead of the infectious force given by (1.4), in this paper, we incorporate the infectious force form (1.2) (when $l=1, h=2$ ) into model (1.3). Our aim is to investigate the psychological effect of certain serious diseases on the community when the ratio of the number of infectives to that of the susceptibles is getting larger. As we can see from the following section, the model is not well defined at the origin and thus it cannot be linearized at it. We want to explore if it can produce some new dynamical behaviors different from the classical epidemic models.

This paper is organized as follows: in Section 2, we present the model and its simplified form. In Section 3, we carry out a qualitative analysis of the model. The existence of all kinds of equilibria and their stability results are derived. Finally, a brief discussion and some numerical simulations are given in Section 4.

\section{The Model}

Assuming that the infectious force takes the form (1.2) and $l=1, h=2$, that is,

$$
g(I, S)=g\left(\frac{I}{S}\right)=\frac{k(I / S)}{1+\alpha(I / S)^{2}}=\frac{k I S}{S^{2}+I^{2}}
$$

consider the following SIRS model:

$$
\begin{aligned}
& \frac{d S}{d t}=b-d S-\frac{k I S^{2}}{S^{2}+\alpha I^{2}}+\gamma R, \\
& \frac{d I}{d t}=\frac{k I S^{2}}{S^{2}+\alpha I^{2}}-(d+\mu) I, \\
& \frac{d R}{d t}=\mu I-(d+\gamma) R .
\end{aligned}
$$

All the parameters are positive and have the similar biological meaning as in (1.3). Noting that system $(2.2)$ is not well defined at the origin $(0,0,0)$, we redefine that when $(S, I, R)=$ $(0,0,0)$,

$$
\frac{d S}{d t}=b, \quad \frac{d I}{d t}=0, \quad \frac{d R}{d t}=0
$$

With this assumption, it is easy to see that the first octant $R_{+}^{3}=\{(S, I, R) \mid S, I, R \geq 0\}$ is positively invariant for system (2.2), and that system (2.2) is continuous and satisfies the 
Lipschitz condition in $R_{+}^{3}$. The following result shows that the solutions of system (2.2) are bounded, and hence, lie in a compact set and are continuable for all positive time.

Lemma 2.1. The plane $S+I+R=b / d$ is an invariant manifold of system (2.2), which is attracting in the first octant.

Proof. Summing up the three equations in (2.2) and denoting

$$
N(t)=S(t)+I(t)+R(t)
$$

we have

$$
\frac{d N}{d t}=b-d N
$$

It is clear that $N(t)=b / d$ is a solution of (2.5) and for any $N\left(t_{0}\right) \geq 0$, the general solution of (2.5) is

$$
N(t)=\frac{1}{d}\left[b-\left(b-d N\left(t_{0}\right)\right) e^{-d\left(t-t_{0}\right)}\right]
$$

Thus,

$$
\lim _{t \rightarrow \infty} N(t)=\frac{b}{d^{\prime}}
$$

which implies the conclusion.

In the following, we consider the existence of equilibria of system (2.2). For any values of parameters, model (2.2) always has a disease-free equilibrium $E_{3}^{0}=(b / d, 0,0)$. To find the positive equilibria, set

$$
\begin{gathered}
b-d S-\frac{k I S^{2}}{S^{2}+\alpha I^{2}}+\gamma R=0, \\
\frac{k S^{2}}{S^{2}+\alpha I^{2}}-(d+\mu)=0, \\
\mu I-(d+\gamma) R=0 .
\end{gathered}
$$

Define the basic reproduction number as follows:

$$
R_{0}=\frac{k}{d+\mu} .
$$

From (2.8), we have

$$
\left[\alpha-\left(R_{0}-1\right)\left(1+\frac{\mu}{d+\gamma}\right)^{2}\right] I^{2}+\frac{2 b}{d}\left(R_{0}-1\right)\left(1+\frac{\mu}{d+\gamma}\right) I-\frac{b^{2}}{d^{2}}\left(R_{0}-1\right)=0 .
$$


It is easy to see from (2.10) that

(i) if $R_{0} \leq 1$, then there is no positive equilibrium;

(ii) if $R_{0}>1$, then there is a unique positive equilibrium $E_{3}^{*}=\left(S^{*}, I^{*}, R^{*}\right)$, called the endemic equilibrium and given by

$$
\begin{aligned}
S^{*} & =\sqrt{\frac{\alpha}{R_{0}-1} I^{*},} \\
I^{*} & =\frac{1}{\sqrt{\alpha /\left(R_{0}-1\right)}+(1+\mu /(d+\gamma))} \frac{b}{d}, \\
R^{*} & =\frac{\mu}{d+\gamma} I^{*} .
\end{aligned}
$$

In the following section, we will study the properties of these equilibria and perform a global qualitative analysis of model (2.2).

\section{Mathematical Analysis}

It is clear that the limit set of system (2.2)-(2.3) is on the plane $S+I+R=b / d$. Thus, we focus on the reduced system:

$$
\begin{aligned}
& \frac{d S}{d t}=\left(b+\frac{\gamma b}{d}\right)-(d+\gamma) S-\gamma I-\frac{k I S^{2}}{S^{2}+\alpha I^{2}}, \\
& \frac{d I}{d t}=\frac{k I S^{2}}{S^{2}+\alpha I^{2}}-(d+\mu) I, \\
& \frac{d S}{d t}=b\left(1+\frac{\gamma}{d}\right), \quad \frac{d I}{d t}=0, \quad \text { when }(S, I)=(0,0),
\end{aligned}
$$

confined to the set

$$
\mathfrak{D}_{S I}=\left\{(S, I) \mid S, I \geq 0, S+I \leq \frac{b}{d}\right\}
$$

To be concise in notations, rescale (3.1) by

$$
S=\frac{(b+\gamma b / d) x}{d+\mu}, \quad I=\frac{(b+\gamma b / d) y}{\gamma}, \quad t=\frac{\tau}{d+\mu},
$$


and, for simplicity, we still use variable $t$ instead of $\tau$. Then, we obtain

$$
\begin{aligned}
& \frac{d x}{d t}=1-q x-y-\frac{a x^{2} y}{x^{2}+p y^{2}}, \\
& \frac{d y}{d t}=\left[\frac{R_{0} x^{2}}{x^{2}+p y^{2}}-1\right] y, \\
& \frac{d x}{d t}=1, \quad \frac{d y}{d t}=0, \quad \text { when }(x, y)=(0,0),
\end{aligned}
$$

where

$$
q=\frac{d+r}{d+\mu}, \quad p=\frac{\alpha(d+\mu)^{2}}{r^{2}}, \quad a=\frac{k}{r}
$$

Denote

$$
\mathfrak{D}_{x y}=\left\{(x, y) \mid x, y \geq 0, x+\frac{a}{R_{0}} y \leq \frac{1}{q}\right\} .
$$

Obviously, $\mathfrak{D}_{x y}$ is a positively invariant set for system (3.4) and every solution initiated from the outside of $\mathfrak{D}_{x y}$ will approach or enter into and stay in it forever as $t$ is getting larger.

Note that the equilibrium $E_{2}^{0}=(1 / q, 0)$ corresponds to the disease-free equilibrium $E_{3}^{0}$ and the unique positive equilibrium $E_{2}^{*}=\left(x^{*}, y^{*}\right)$ corresponding to the endemic equilibrium $E_{3}^{*}$ exists if and only if the condition $R_{0}>1$, where

$$
x^{*}=\frac{1}{\left(1+a / R_{0}\right) \sqrt{\left(R_{0}-1\right) / p}+q}, \quad y^{*}=\sqrt{\frac{R_{0}-1}{p}} x^{*} .
$$

Note that we are interested in the dynamics of system (3.4) in the interior of the first quadrant. Thus, we can make a time scale change:

$$
d t=\left(x^{2}+p y^{2}\right) d \tau
$$

such that system (3.4) is equivalent to the following system in the interior of the first quadrant. For simplicity, we still use variable $t$ instead of $\tau$ :

$$
\begin{aligned}
& \frac{d x}{d t}=x^{2}+p y^{2}+\Phi(x, y) \equiv P(x, y), \\
& \frac{d y}{d t}=\Psi(x, y) \equiv Q(x, y),
\end{aligned}
$$

where

$$
\begin{aligned}
& \Phi(x, y)=-q x^{3}-(a+1) x^{2} y-p q x y^{2}-p y^{3} \\
& \Psi(x, y)=\left(R_{0}-1\right) x^{2} y-p y^{3} .
\end{aligned}
$$


We should point out that except for the equilibria $E_{2}^{0}$ and $E_{2}^{*}$, system (3.9) has another more equilibrium $\bar{E}_{2}^{0}=(0,0)$ than (3.4) since the time scaling is (3.8).

In the following, we will study the properties of the equilibria and perform a global qualitative analysis of model (3.9).

\subsection{Asymptotic Behavior of the System (3.9) at $\bar{E}_{2}^{0}=(0,0)$}

The equilibrium $\bar{E}_{2}^{0}$ of system (3.9) is an isolated critical point of higher order. Obviously, system (3.9) is analytic in a neighborhood of the origin. By [14, Theorem 3.10 on page 79], any orbit of (3.9) tending to the origin must tend to it spirally or along a fixed direction, which depends on the characteristic equation of system (3.9). Here, we will show that if a solution orbit of (3.9) tends to the origin, then it must tend to it along a fixed direction. We will also determine the number of solution orbits of system (3.9) that tend to $\bar{E}_{2}^{0}$ along a fixed direction as $t \rightarrow+\infty$ or $t \rightarrow-\infty$ in the interior of the first quadrant by using the results in [14]. Hereafter, we refer to [14] for results and explanations of several notations involved.

First of all, we introduce the polar coordinates $x=r \cos \theta, y=r \sin \theta$ and define

$$
G(\theta)=-\sin \theta X_{2}(\cos \theta, \sin \theta),
$$

where $X_{2}(x, y)$ is homogeneous polynomial in $x$ and $y$ of degree 2 in the first equation of (3.9). Then the characteristic equation of system (3.9) takes the form

$$
G(\theta)=-\sin \theta\left(\cos ^{2} \theta+p \sin ^{2} \theta\right)=0 .
$$

Clearly, $G(\theta)=0$ has two roots $\theta_{0}=0$ and $\theta_{1}=\pi$. By the results in [14, section II.2], we know that no orbit of system (3.9) can tend to the critical point $\bar{E}_{2}^{0}$ spirally, and any orbit of system (3.9) approaching the origin must be along the directions $\theta_{0}=0$ or $\theta_{1}=\pi$. Since we are only interested in the interior of the first octant, we just consider the direction $\theta_{0}=0$.

To determine if there exists an orbit of system (3.9) which tends to the origin along the direction $\theta_{0}=0$ as $t$ tends to $+\infty$ or $-\infty$, we have to compute the derivative of $G(\theta)$ and the function $H(\theta)$ :

$$
\begin{aligned}
& G^{\prime}(\theta)=-\cos ^{3} \theta+(2-3 p) \sin ^{2} \theta \cos \theta, \\
& H(\theta)=\cos \theta\left(\cos ^{2} \theta+p \sin ^{2} \theta\right) .
\end{aligned}
$$

Theorem 3.1. There exist $\varepsilon>0$ and $r_{0}>0$ such that there exists a unique orbit of system (3.9) in the sector $\left\{(\theta, r): 0 \leq \theta<\varepsilon, 0<r<r_{0}\right\}$ that tends to $\bar{E}_{2}^{0}$ along $\theta_{0}=0$ as $t \rightarrow-\infty$.

Proof. Since $G^{\prime}\left(\theta_{0}\right)=-1$ and $H\left(\theta_{0}\right)=1$, by [14, Theorem 3.7 on page 70], there exist $\varepsilon>0$ and $r_{0}>0$ such that there exists a unique orbit of system (3.9) in the sector $\{(\theta, r): 0 \leq \theta<\varepsilon, 0<$ $\left.r<r_{0}\right\}$ that tends to $\bar{E}_{2}^{0}$ along $\theta_{0}=0$ as $t \rightarrow-\infty$. The proof is thus completed.

In fact, the only orbit tending to $\bar{E}_{2}^{0}$ along $\theta_{0}=0$ as $t \rightarrow-\infty$ is $y=0$. 


\subsection{The Disease-Free Equilibrium $E_{2}^{0}=(1 / q, 0)$ of System (3.9)}

First,we give the following result regarding the nonexistence of periodic orbits in system (3.9), which implies the nonexistence of periodic orbits of system (2.2) by Lemma 2.1.

Theorem 3.2. System (3.9) does not have nontrivial periodic orbits.

Proof. Consider system (3.9) for $x>0$ and $y>0$. Take a Dulac function

$$
D(x, y)=\frac{1}{y\left(x^{2}+p y^{2}\right)}
$$

We have

$$
\frac{\partial(D P)}{\partial x}+\frac{\partial(D Q)}{\partial y}=\frac{-q x^{4} y-2 p q x^{2} y^{3}-2 a p x y^{4}-p^{2} q y^{5}}{y^{2}\left(x^{2}+p y^{2}\right)^{2}}-\frac{2 R_{0} p x^{2} y}{\left(x^{2}+p y^{2}\right)^{2}}<0 .
$$

The conclusion follows. $(1 / q, 0)$.

The following theorem shows the properties of the disease-free equilibrium $E_{2}^{0}=$

Theorem 3.3. The disease-free equilibrium $E_{2}^{0}$ of system (3.9) is

(i) a global asymptotically stable node if $R_{0}<1$;

(ii) a saddle if $R_{0}>1$;

(iii) global asymptotically stable if $R_{0}=1$.

Proof. The Jacobian matrix of system (3.9) at $E_{2}^{0}$ is

$$
A=\left(\begin{array}{cc}
-\frac{1}{q} & -\frac{a+1}{q^{2}} \\
0 & \frac{R_{0}-1}{q^{2}}
\end{array}\right),
$$

whose determinant and trace are, respectively,

$$
\operatorname{det} A=\frac{1-R_{0}}{q^{3}}, \quad \operatorname{tr} A=\frac{R_{0}-1-q}{q^{2}} .
$$

However $E_{2}^{0}$ is locally stable (unstable) if the trace $\operatorname{tr} A<0(\operatorname{tr} A>0)$ and $\operatorname{det} A>0$.

If $R_{0}<1$, we have $\operatorname{det} A>0$ and $\operatorname{tr} A<0$. Hence, $E_{2}^{0}$ is locally asymptotically stable. Note that the interior equilibrium does not exist and $\bar{E}_{2}^{0}$ is unstable to the positive quadrant. By Theorem 3.2, the disease-free equilibrium $E_{2}^{0}$ is global asymptotically stable.

If $R_{0}>1$, we have $\operatorname{det} A<0$. So $E_{2}^{0}$ is a saddle. 
If $R_{0}=1$, system (3.9) becomes

$$
\begin{aligned}
& \frac{d x}{d t}=x^{2}+p y^{2}-q x^{3}-(a+1) x^{2} y-p q x y^{2}-p y^{3} \\
& \frac{d y}{d t}=-p y^{3}
\end{aligned}
$$

Translating the boundary equilibrium $E_{2}^{0}$ of system (3.18) to the origin, system (3.18) can be written as

$$
\begin{aligned}
& \frac{d x}{d t}=-\frac{x}{q}-\frac{a+1}{q^{2}} y+\bar{\Phi}(x, y), \\
& \frac{d y}{d t}=-p y^{3},
\end{aligned}
$$

where

$$
\bar{\Phi}(x, y)=-2\left(x^{2}+\frac{(a+1)}{q^{2}} x y\right)-\left(q x^{3}+(a+1) x^{2} y+p q x y^{2}+p y^{3}\right) .
$$

In order to study the stability of $(0,0)$ of system $(3.19)$, we firstly carry out a coordinate transformation $u=y, v=(x / q)+\left((a+1) / q^{2}\right) y, d t=q d \tau$, for system (3.19), we obtain

$$
\begin{aligned}
& \frac{d u}{d \tau}=-p u^{3} \\
& \frac{d v}{d \tau}=-v+F(u, v)
\end{aligned}
$$

where $F(u, v)$ is a polynomial in $u$ and $v$ of a degree not less than 2. Obviously, (3.21) has a center manifold $v=h(u)$. Since the solution on the center manifold satisfies

$$
\frac{d u}{d \tau}=-p u^{3}
$$

we know that the origin of (3.22) is asymptotically stable. By the theorem of center manifold, we know that the origin $(0,0)$ of $(3.21)$ is also asymptotically stable. That is to say, the boundary equilibrium $(1 / q, 0)$ of system $(3.18)$ is asymptotically stable. Note that when $R_{0}=1$, system (3.18) has no positive equilibrium and $\bar{E}_{2}^{0}$ is unstable to the positive quadrant. By Theorem 3.2, we obtain that all the orbits of system (3.18) in the first quadrant tend to the equilibrium $E_{2}^{0}$. The proof of Theorem 3.3 is thus completed.

\subsection{The Endemic Equilibrium $E_{2}^{*}=\left(x^{*}, y^{*}\right)$ of System (3.9)}

Theorem 3.4. Suppose $R_{0}>1$, then there is a unique endemic equilibrium $\left(x^{*}, y^{*}\right)$ of $(3.9)$, which is a global asymptotically stable node. 
Proof. The Jacobian matrix of system (3.9) at the equilibrium $\left(x^{*}, y^{*}\right)$ is

$$
A=\left(\begin{array}{cc}
2 x^{*}-3 q\left(x^{*}\right)^{2}-2(a+1) x^{*} y^{*}-p q\left(y^{*}\right)^{2} & 2 p y^{*}-(a+1)\left(x^{*}\right)^{2}-2 p q x^{*} y^{*}-3 p\left(y^{*}\right)^{2} \\
2\left(R_{0}-1\right) x^{*} y^{*} & \left(R_{0}-1\right)\left(x^{*}\right)^{2}-3 p\left(y^{*}\right)^{2}
\end{array}\right)
$$

whose determinant and trace are respectively,

$$
\begin{aligned}
\operatorname{det} A & =6 q R_{0}\left(R_{0}-1\right)\left(x^{*}\right)^{4}+6 p\left(a+R_{0}\right) x^{*}\left(y^{*}\right)^{3}+4 R_{0}\left(1-R_{0}\right)\left(x^{*}\right)^{3} \\
& =\left\{6 R_{0}\left(R_{0}-1\right)\left[q+\left(1+\frac{a}{R_{0}}\right) \sqrt{\frac{R_{0}-1}{p}}\right] x^{*}+4 R_{0}\left(1-R_{0}\right)\right\}\left(x^{*}\right)^{3} \\
& =2 R_{0}\left(R_{0}-1\right)\left(x^{*}\right)^{3}, \\
\operatorname{tr} A & =2 x^{*}-3 q\left(x^{*}\right)^{2}-2(a+1) x^{*} y^{*}-p q\left(y^{*}\right)^{2}+\left(R_{0}-1\right)\left(x^{*}\right)^{2}-3 p\left(y^{*}\right)^{2} \\
& =\left\{\left[2-2 R_{0}-2 q-q R_{0}-2(a+1) \sqrt{\frac{R_{0}-1}{p}}\right] x^{*}+2\right\} x^{*} \\
& =\left[2\left(1-R_{0}\right)-q R_{0}-2 a \sqrt{\frac{R_{0}-1}{p}}\left(1-\frac{1}{R_{0}}\right)\right]\left(x^{*}\right)^{2} .
\end{aligned}
$$

Since $R_{0}>1$, we have $\operatorname{det} A>0$ and $\operatorname{tr} A<0$. Hence, $\left(x^{*}, y^{*}\right)$ is locally asymptotically stable. Note that $\bar{E}_{2}^{0}$ is unstable to the interior of the positive quadrant, and that the two stable manifolds of $E_{2}^{0}$ are on the $x$-axis and one of its unstable manifolds points to the the positive quadrant. By Theorem 3.2, we know that the interior equilibrium $\left(x^{*}, y^{*}\right)$ of system (3.9) is a global asymptotically stable node. This proves the theorem.

Summarizing Theorems 3.1-3.4, we have the following results on the dynamics of model (3.1).

Theorem 3.5. Let $R_{0}=k /(d+\mu)$, then the following hold.

(i) If $R_{0}<1$, then model (3.1) has a unique disease-free equilibrium $(b / d, 0)$, which is a global attractor in the first quadrant (see Figure 1).

(ii) If $R_{0}=1$, then mode (3.1) has a unique disease-free equilibrium $(b / d, 0)$, which attracts all orbits in the interior of the first quadrant (see Figure 2).

(iii) If $R_{0}>1$, then model (3.1) has two equilibria, a disease-free equilibrium $(b / d, 0)$ and an endemic equilibrium $\left(x^{*}, y^{*}\right)$. The endemic equilibrium is a global attractor in the interior of the first quadrant (see Figure 3). 


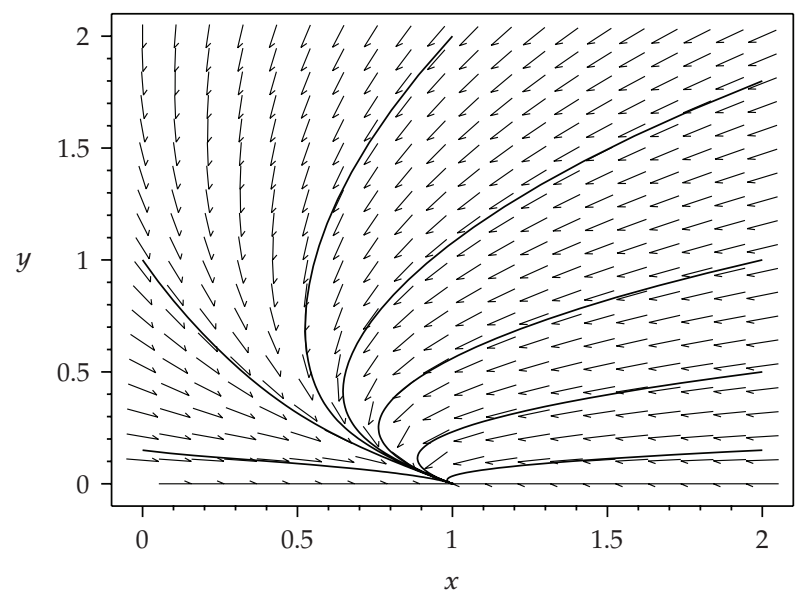

Figure 1: Topological structure of system (3.1) at $(b / d, 0)$ when $R_{0}<1$, where $b=1, d=1, k=1, \gamma=$ $1, \alpha=1, \mu=1$.

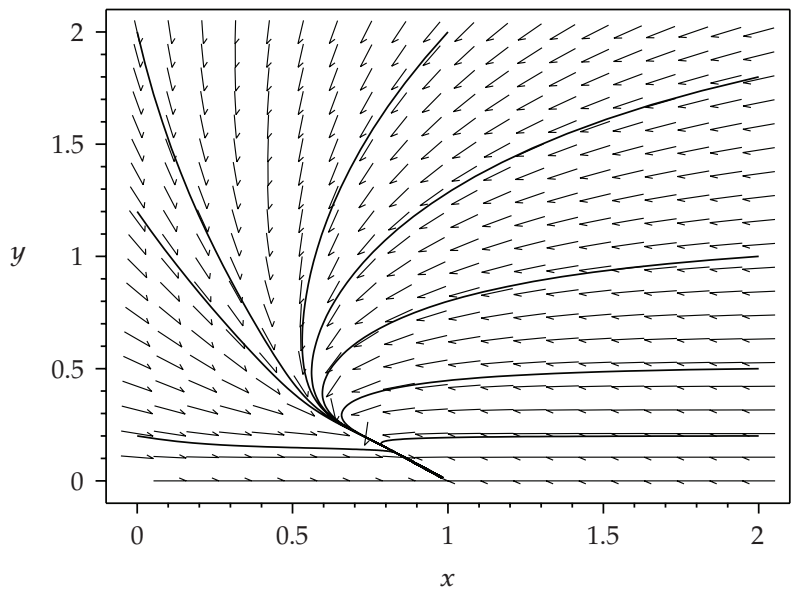

Figure 2: Topological structure of system (3.1) at $(b / d, 0)$ when $R_{0}=1$, where $b=1, d=1, k=1.5, \gamma=$ $0.5, \alpha=1, \mu=0.5$.

\section{Discussion}

Several nonlinear incidence rates have been proposed by researchers, see, for example, Capasso and Serio [4], Liu et al. [6], Derrick and van den Driessche [7], Hethcote and van den Driessche [10], and so forth Complex dynamics have been observed in epidemiological models with nonlinear incidence rate, such as the existence of multiple equilibria and limit cycles, various types of bifurcations including Hopf, saddle-node, homoclinic, and Bagdanov-Takens bifurcations, see also Ruan and Wang [12] and references cited therein.

In this paper, different from the classical nonlinear incident rate, we assume that the infectious force is a function of the ratio of the number of the infectives to that of the susceptibles which takes the form $k I S /\left(S^{2}+\alpha I^{2}\right)$. Our aim is to investigate the psychological effect of certain serious diseases on the community when the ratio of the number of infectives to that of the susceptibles is getting larger. Note that the model with such infectious force 


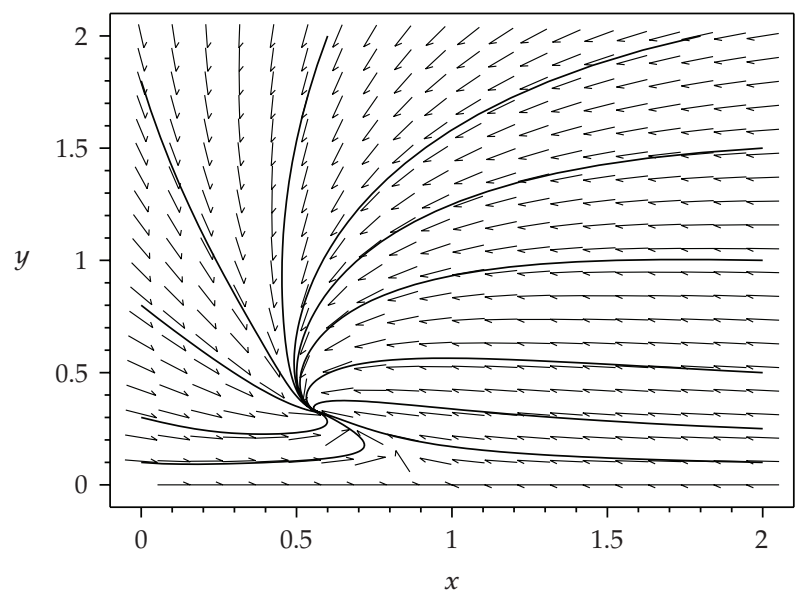

Figure 3: Topological structure of system (3.1) at $(b / d, 0)$ and $\left(x^{*}, y^{*}\right)$ when $R_{0}>1$, where $b=1, d=1, k=$ $2, \gamma=0.5, \alpha=1, \mu=0.5$.

is not well defined at the origin. By redefining system (2.2) at the origin $(0,0,0)$, reducing it on the invariant manifold $S+I+R=b / d$, we get system (3.1). For system (3.1), after simplification and transformation in the time variable, we get its equivalent polynomial system (3.9). Notice that (3.9) has another more equilibrium $\bar{E}_{2}^{0}=(0,0)$ than (3.4) since the time scaling is (3.8). Qualitative study on the the model shows that the model does not exhibit complicated dynamics as other epidemic models with other types of incidence rates reported by Liu et al. [6], Derrick and van den Driessche [7], Hethcote and Levin [9], Hethcote and van den Driessche [10], and Ruan and Wang [12], We observe that if the positive equilibrium of the system is locally asymptotically stable, then the system does not have any nontrivial positive periodic solutions. Numerical simulations illustrate our results.

\section{Acknowledgment}

Research is supported by the National Natural Science Foundation of China (10871129) and the Educational Committee Foundation of Shanghai (09YZ208).

\section{References}

[1] V. Capasso, Mathematical Structure of Epidemic System, vol. 97 of Lecture Note in Biomathematics, Springer, Berlin, Germany, 1993.

[2] S. A. Levin, T. G. Hallam, and L. J. Gross, Applied Mathematical Ecology, vol. 18 of Biomathematics, Springer, Berlin, Germany, 1989.

[3] J. Cui, Y. Sun, and H. Zhu, "The impact of media on the control of infectious diseases," Journal of Dynamics and Differential Equations, vol. 20, no. 1, pp. 31-53, 2008.

[4] V. Capasso and G. Serio, "A generalization of the Kermack-McKendrick deterministic epidemic model," Mathematical Biosciences, vol. 42, no. 1-2, pp. 43-61, 1978.

[5] W. M. Liu, H. W. Hethcote, and S. A. Levin, "Dynamical behavior of epidemiological models with nonlinear incidence rates," Journal of Mathematical Biology, vol. 25, no. 4, pp. 359-380, 1987.

[6] W. M. Liu, S. A. Levin, and Y. Iwasa, "Influence of nonlinear incidence rates upon the behavior of SIRS epidemiological models," Journal of Mathematical Biology, vol. 23, no. 2, pp. 187-204, 1986. 
[7] W. R. Derrick and P. van den Driessche, "A disease transmission model in a nonconstant population," Journal of Mathematical Biology, vol. 31, no. 5, pp. 495-512, 1993.

[8] H. W. Hethcote, "The mathematics of infectious diseases," SIAM Review, vol. 42, no. 4, pp. 599-653, 2000.

[9] H. W. Hethcote and S. A. Levin, "Periodicity in epidemiological models," in Applied Mathematical Ecology, L. Gross, T. G. Hallam, and S. A. Levin, Eds., vol. 18 of Biomathematics, pp. 193-211, Springer, Berlin, Germany, 1989.

[10] H. W. Hethcote and P. van den Driessche, "Some epidemiological models with nonlinear incidence," Journal of Mathematical Biology, vol. 29, no. 3, pp. 271-287, 1991.

[11] M. E. Alexander and S. M. Moghadas, "Periodicity in an epidemic model with a generalized nonlinear incidence," Mathematical Biosciences, vol. 189, no. 1, pp. 75-96, 2004.

[12] S. Ruan and W. Wang, "Dynamical behavior of an epidemic model with a nonlinear incidence rate," Journal of Differential Equations, vol. 188, no. 1, pp. 135-163, 2003.

[13] D. Xiao and S. Ruan, "Global analysis of an epidemic model with nonmonotone incidence rate," Mathematical Biosciences, vol. 208, no. 2, pp. 419-429, 2007.

[14] Z. F. Zhang, T. R. Ding, W. Z. Huang, and Z. X. Dong, Qualitative Theory of Differential Equations, vol. 101 of Translations of Mathematical Monographs, American Mathematical Society, Providence, RI, USA, 1992. 


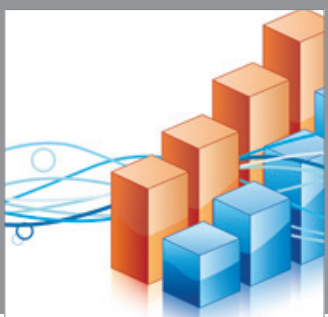

Advances in

Operations Research

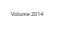

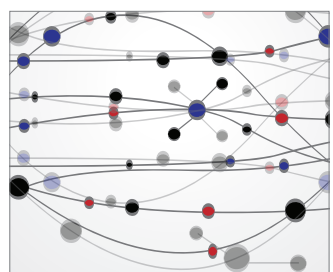

\section{The Scientific} World Journal
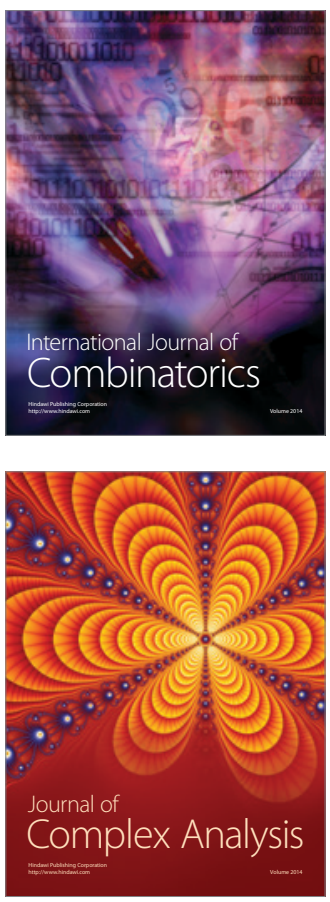

International Journal of

Mathematics and

Mathematical

Sciences
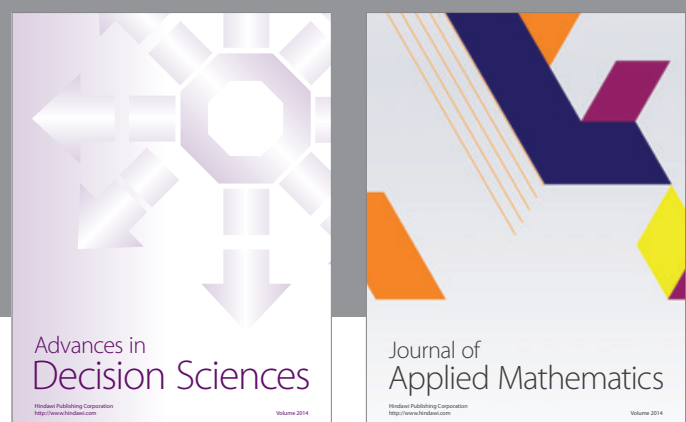

Journal of

Applied Mathematics
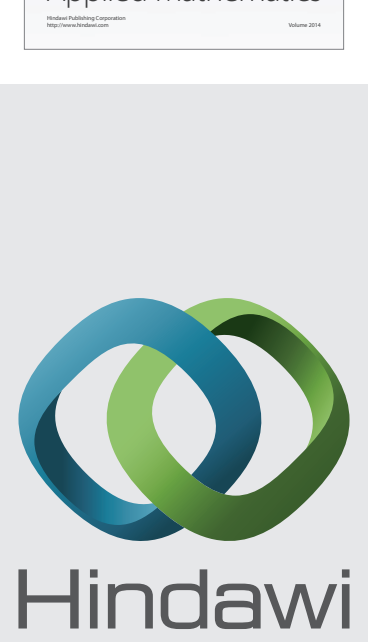

Submit your manuscripts at http://www.hindawi.com
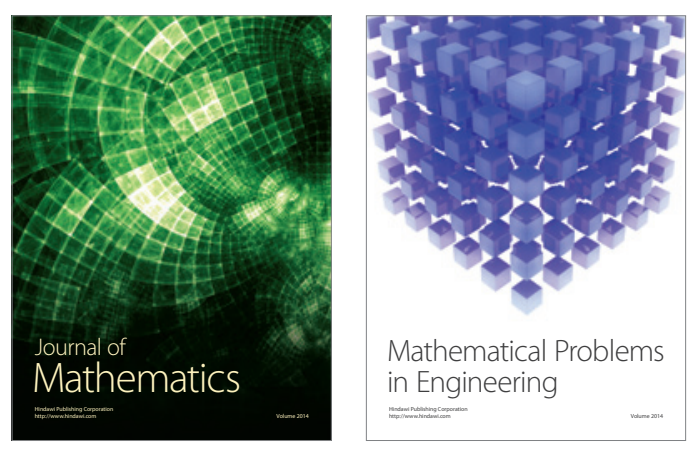

Mathematical Problems in Engineering
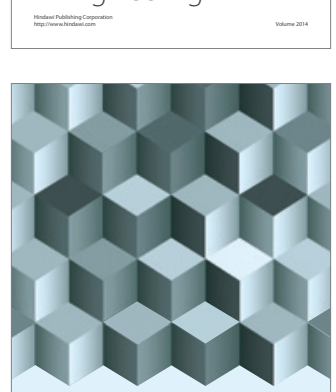

Journal of

Function Spaces
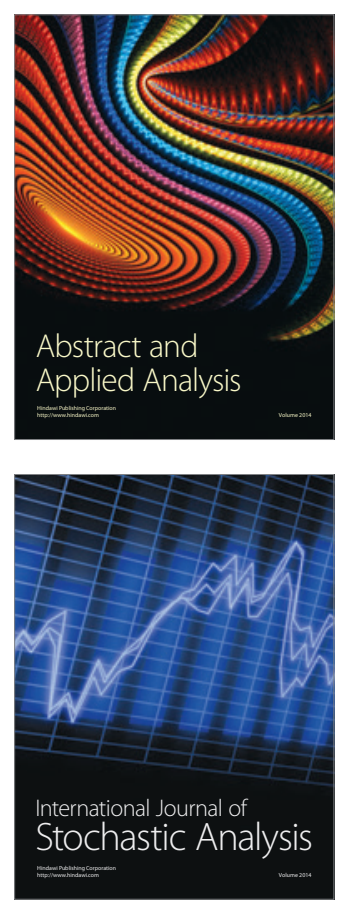

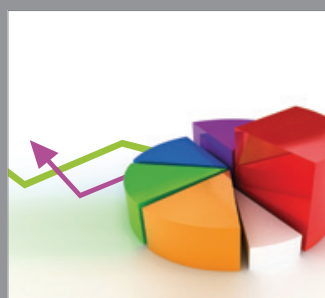

ournal of

Probability and Statistics

Promensencen
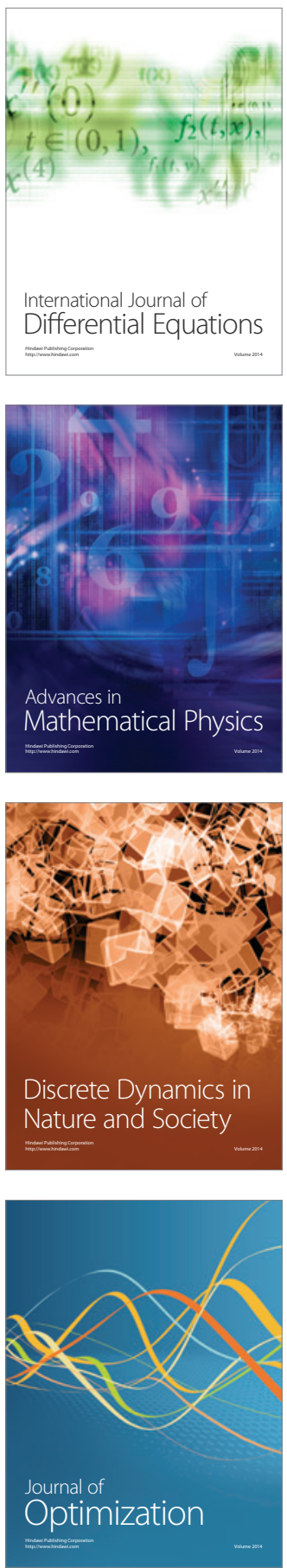